\title{
The Effect of TCM Herbs on Mitochondrial Functions: The Linkage between $Q i$ and Mitochondria
}

\author{
Yu Chen ${ }^{*}$, Qian Feng1*, Mengmei Li ${ }^{1}$, Zhongzhen Cai1, Yuming Chen ${ }^{1}$, Lin Wang1, Jie Teng1, \\ Yanjie Chen ${ }^{1}$, Wenjun Wang1, Glen Rein ${ }^{2}$, Bruce Qing Tang ${ }^{1}$, Xuemei Bai ${ }^{1 \#}$
}

${ }^{1}$ ENNOVA Institute of Life Science and Technology, ENN Group, Langfang, China

${ }^{2}$ Quantum Biology Research Lab, Ridgway, USA

Email: \#baixuemei@enn.cn

How to cite this paper: Chen, Y., Feng, Q., Li, M.M., Cai, Z.Z., Chen, Y.M., Wang, L., Teng, J., Chen, Y.J., Wang, W.J., Rein, G., Tang, B.Q. and Bai, X.M. (2020) The Effect of TCM Herbs on Mitochondrial Functions: The Linkage between Qi and Mitochondria. Chinese Medicine, 11, 127-141. https://doi.org/10.4236/cm.2020.114008

Received: November 1, 2020

Accepted: December 27, 2020

Published: December 30, 2020

Copyright $\odot 2020$ by author(s) and Scientific Research Publishing Inc. This work is licensed under the Creative Commons Attribution International License (CC BY 4.0).

http://creativecommons.org/licenses/by/4.0/ (c) (i) Open Access

\begin{abstract}
The linkage between Qi and mitochondria was investigated by exploring the effect of Traditional Chinese Medicine (TCM) Qi-invigorating herbs on mitochondrial function at the biochemical and molecular levels. Three Chinese herbs (Astragali radix, Herba cistanche, Panax ginseng) were used to treat cultured mouse kidney cells and the generation of adenosine triphosphate (ATP) was measured. The Qi-invigorating herb, Astragali radix, was selected for further study using additional biological and molecular parameters, including ATP, reactive oxygen species (ROS), mitochondrial membrane potential (MMP), mtDNA copies, superoxide dismutase (SOD), glutathione (GSH), cell growth, cell viability and transcriptomes. We also chose two concentrations of Astragali radix to study the hormetic effect. The results indicated that: 1) Qi-invigorating herbs have significant effects on the function of mitochondria, with ATP production and the antioxidant capacity being significantly enhanced, and ROS levels being reduced, allowing for a more optimal oxidation environment. The effect of the herbs followed a hormetic curve with a stimulating effect at lower doses but an inhibiting effect at high doses; 2) The growth of the cells was not affected despite numerous biochemical changes associated with mitochondrial function, indicating the powerful ability of mitochondria to maintain cellular homeostasis; 3) The up-regulation of NOCT gene, related to nicotinamide adenine dinucleotide (NADH) synthesis, offers a molecular basis for the ATP-promoting effect of the Qi-invigorating herbs. This work provides additional insight into the efficacy of TCM herbs from a western perspective.
\end{abstract}

\section{Keywords}

Qi-Invigorating Herbs, Qi, Mitochondria, Hormesis

${ }^{\star}$ The authors Yu Chen and Qian Feng contributed equally to the work. ${ }^{\#}$ Corresponding author. 


\section{Introduction}

As mankind is facing more and more challenges of different types of diseases, it calls on all medical knowledge accumulated throughout human history to combat current epidemics. Traditional Chinese Medicine (TCM) and western medicine are two very different systems and the integration of these two systems will help advance contemporary medical practices to better address these challenges.

In TCM, Qi (Prana, Chi, vitality) is the single most important concept, which stimulates the flow of blood throughout the body, and promotes the absorption and utility of the nutrients of food [1]. However, Qi still cannot be measured by modern technology by now. Mitochondria are considered to be the engine of life with the main function of providing ATP for cellular activities through oxidative phosphorylation [2]. Qi in the TCM system and mitochondria in modern medicine system play similar roles in providing power for cells and promoting the transformation of material and energy [3], and therefore, a connection between the mitochondria and Qi has been suggested [4] [5] [6]. In addition, many researchers have proposed that there is a great deal of similarity in their characteristics and functions between Qi and mitochondria [7] [8] [9].

In recent years, the study of mitochondria has gained more and more interest in medicine because numerous diseases have been attributed to mitochondrial dysfunction [10] [11] [12]. In addition, compelling evidence supports the critical role that mitochondria play in maintaining homeostasis under environmental stress [13]. However, so far, this linkage is not fully examined and supported by scientific evidence. Therefore, obtaining such evidence is of great importance as it will help to bridge the gap between TCM and western medicine.

Given the difficulty in studying Qi, we utilized Qi-invigorating herbs as the carrier of Qi for this study. Qi-invigorating herbs have been widely used in TCM under various conditions including coronary heart disease [14] [15] [16], treatment of Helicobacter pylori (Hp), chronic gastritis of the spleen and stomach [17], gastric cancer [18] [19] and postoperative recurrence of ovarian endometrial cysts [20]. However, the biochemical mechanism for these herbs is not well understood. The Yin-Yang theory is vital to the conceptual system of TCM. According to the theory, Yin and Yang, the two complementary, but opposing, internal forces, govern the functions of the human body [21]. Studies conducted by Ko et al. have shown that the Yang-invigorating and Yin-invigorating herbs have different impacts on mitochondrial function, with Yang-herbs enhancing ATP generation, and Yin-herbs increasing the immunomodulatory function of cells [22] [23] [24] [25]. Qi-invigorating herbs fall into the category of the Yang group, with some enhancing ATP generation and others suppressing it [9].

For this study, we utilized mouse renal collecting duct cells (M-1 cells) to examine the effect of TCM herbs on mitochondrial functions including ATP production, reactive oxygen species (ROS), Mitochondrial membrane potential (MMP), and mtDNA copies. To better understand the molecular mechanisms, we also conducted an analysis of the transcriptome, with the goal of providing more 
scientific evidence of the linkage between Qi-invigorating herbs and mitochondrial function. Such evidence will help to understand the nature of Qi from a western medicine perspective, which will help bridge the gap between TCM and western medicine.

\section{Materials and Methods}

\subsection{Herbal Preparation}

All herbal materials were purchased from a Chinese medicine store (Tongrentang chain-store), and the herbal preparations were prepared as follows: 1) $A s$ tragali radix extraction: Astragali radix (50 g) was cut into small pieces and soaked for an hour in $500 \mathrm{~mL}$ deionized water and then extracted twice by boiling water for 30 min each time. The combined extract was dried by evaporation under reduced pressure to obtain an aqueous extract of Astragali radix with a yield of $14.4 \%(\mathrm{w} / \mathrm{w})$; 2) Herba cistanche extraction: Herba cistanche (100 g) was cut into small pieces and then extracted by heating under reflux in $300 \mathrm{~mL} 95 \%$ ethanol at $65^{\circ} \mathrm{C}$ for $2 \mathrm{~h}$. The procedure was repeated twice. The pooled extract was dried by evaporating the solvent under reduced pressure to obtain an aqueous extract of Herba cistanche with a yield of $13.2 \%$ (w/w); 3) Panax ginseng extraction: Panax ginseng ( $100 \mathrm{~g})$ was cut into small pieces and then extracted by heating under reflux in $500 \mathrm{~mL} 95 \%$ ethanol at $65^{\circ} \mathrm{C}$ for $2 \mathrm{~h}$. The procedure was repeated twice. The pooled extract was dried by evaporating the solvent under reduced pressure to obtain an ethanol extract of Panax ginseng with a yield of $5 \%(\mathrm{w} / \mathrm{w})$.

\subsection{Cell Culture}

For these experiments, M-1 renal collecting duct cells were obtained from National Infrastructure of Cell Line Resource (Beijing, China, http://www.cellresource.cn). The cells were cultured as monolayers in Dulbecco's modified Eagle's medium (DMEM) supplemented with $10 \%$ fetal bovine serum (FBS) and incubated in a humidified $5 \% \mathrm{CO}_{2}$ incubator at $37^{\circ} \mathrm{C}$. The medium was changed $2-3$ times per week. Stock cells were grown in a $75 \mathrm{~mL}$ culture flask and split at a sub-cultivation ratio of 1:5.

\subsection{Experimental Design}

1) Comparison of different herbal treatment

For these experiments, M-1 cells were seeded at a density of $2 \times 10^{4}$ cells/well into 6-well plates. After cell attachment for $24 \mathrm{~h}$, cells for the experimental group were treated with different herbal extracts (dissolved in phosphate-buffered saline; PBS) at different concentrations $(12.5-200 \mu \mathrm{g} / \mathrm{mL})$ for $4 \mathrm{~h}$. The untreated control group was given PBS only. After the treatment period, the cells were collected using trypsin-EDTA $(0.25 \%)$, centrifuged at $800 \mathrm{~g}$ for $5 \mathrm{~min}$ and re-suspended in $1 \mathrm{~mL}$ of PBS (pH7.4) for ATP assays. All experiments were performed in triplicates. 


\section{2) Effect of Astragali radix at different doses}

M-1 cells were seeded at a density of $5-6 \times 10^{5}$ cells/plate into $10 \mathrm{~cm}$ plates. After cell attachment for $24 \mathrm{~h}$, for the treatment group, the Astragali radix extract (dissolved in phosphate-buffered saline; PBS) was added to the medium. Cells were treated with two final concentrations of $50 \mu \mathrm{g} / \mathrm{mL}$ (low dose) and 250 $\mu \mathrm{g} / \mathrm{mL}$ (high dose). After $4 \mathrm{~h}$ of treatment, the culture medium containing the herbal extracts was removed and replaced with fresh medium. Cells continued to grow with samples being collected at $4 \mathrm{~h}, 24 \mathrm{~h}$ and $48 \mathrm{~h}$. Cells were treated with trypsin-EDTA $(0.25 \%)$, followed by centrifugation at $800 \mathrm{~g}$ for $5 \mathrm{~min}$ before re-suspension in $5 \mathrm{~mL}$ PBS for the different assays. All experiments were performed in triplicate.

\subsection{Biochemical Analysis}

1) ATP assay

The new HPLC method of Chen et al was employed to measure the ATP levels in M-1 cells [26]. An aliquot of $1 \mathrm{~mL}$ cell samples was centrifuged at 10,000 $\mathrm{g}$ for 5 min to collect cells, followed by adding $100 \mu \mathrm{L}$ PBS and $40 \mu \mathrm{L}$ deionized water successively to rupture the cells. Then, $360 \mu \mathrm{L}$ perchloric acid (6\%) was added to remove the protein by keeping samples on ice for an additional $10 \mathrm{~min}$. The cell extract was centrifuged at $10,000 \mathrm{~g}$ at $4^{\circ} \mathrm{C}$ for $5 \mathrm{~min}$, with $300 \mu \mathrm{L}$ of the supernatant neutralized with $40 \mu \mathrm{L}$ of $2 \mathrm{M} \mathrm{K}_{2} \mathrm{CO}_{3}$ and filtered through a $0.45 \mu \mathrm{m}$ filter. Neutralized cell extract $(10 \mu \mathrm{L})$ was used for determination of ATP, which was carried out using HPLC with a mobile phase consisting of $0.1 \mathrm{M} \mathrm{KH}_{2} \mathrm{PO}_{4}$ buffer, pH 6.25 and $5 \%$ methanol $(\mathrm{v} / \mathrm{v})$ set at a rate of $0.6 \mathrm{~mL} / \mathrm{min}$ and a detection wavelength of $254 \mathrm{~nm}$. ATP quantitation was calculated by computing its peak area with identification and quantification using injections of standard solutions of ATP with known concentrations. Total ATP levels in cell homogenate were normalized to the total cell protein.

\section{2) Mitochondrial ROS determination}

ROS levels were quantified in accordance with the protocol of the Reactive Oxygen Species Assay Kit (Product No.: S0033; Beyotime, China). An aliquot of $0.5 \mathrm{~mL}$ cell samples was incubated with DCFH-DA solution $(2 \mu \mathrm{M})$ at $37^{\circ} \mathrm{C}$ for 40 min in the dark. Samples were then washed twice with PBS for measurement. The fluorescence intensity was recorded at $485 \mathrm{~nm}$ (excitation) and $535 \mathrm{~nm}$ (emission) using a microplate reader (Tecan, Switzerland). Total ROS levels in cells were normalized to the total cell protein.

3) $M M P$ assay

MMP was quantified in accordance with the protocol of the Mitochondrial Membrane Potential Assay Kit (Product No.: C2006; Beyotime, China). An aliquot of $0.5 \mathrm{~mL}$ cell samples was incubated with $\mathrm{JC}-1$ probe at $37^{\circ} \mathrm{C}$ for $20 \mathrm{~min}$ in the dark. After the incubation, cells were washed with ice-cold JC-1 buffer solution twice. The JC-1 probe entering the mitochondrial matrix will aggregate and emit at $590 \mathrm{~nm}$ (red) upon excitation at $525 \mathrm{~nm}$, whereas the monomeric JC-1 remaining in the cytoplasm emits at $530 \mathrm{~nm}$ (green) upon excitation at $490 \mathrm{~nm}$. 
The fluorescence intensity was recorded using a microplate reader (Tecan, Switzerland), with the value of MMP calculated by the ratio of red to green.

4) Protein assay

Cell quantity was evaluated as protein concentration using a protein assay kit (Product No.: P0006C; Beyotime, China) with bovine serum albumin as standard $(0-1.5 \mathrm{mg} / \mathrm{mL})$. An aliquot of $0.5 \mathrm{~mL}$ cell samples was centrifuged at 3,000 $\mathrm{g}$ for $5 \mathrm{~min}$ to collect cells, which was then incubated with Triton $(0.1 \%)$ for 30 $\mathrm{min}$. After cell disruption, samples were centrifuged at $11,000 \mathrm{~g}$ for $5 \mathrm{~min}$ to remove cell residue. The supernatant was used for the optical density measurement at a wavelength of $595 \mathrm{~nm}$ by using a microplate reader (Tecan, Switzerland).

\section{5) Cell viability assay}

Cell viability was evaluated by using the Cell Counting Kit-8 (CCK-8) (Product No.: C0038x; Beyotime, China). briefly, an aliquot of $0.5 \mathrm{~mL}$ cell samples was centrifuged at 3,000 $\mathrm{g}$ for $5 \mathrm{~min}$ to collect the cells, which were then re-suspended in $100 \mu \mathrm{L}$ culture medium and seeded in 96-well plates. Finally, $10 \mu \mathrm{L}$ CCK-8 was added to the wells and the plates were incubated for an additional $3 \mathrm{~h}$. The optical density (OD) was measured at a wavelength of $450 \mathrm{~nm}$ using a microplate reader (Tecan, Switzerland).

\section{6) Antioxidant levels}

Antioxidant levels were evaluated by the enzyme activities of SOD and GSH using an SOD Assay Kit with WST-8 (Product No.: S0101; Beyotime, China) and a total Glutathione Assay Kit (Product No.: S0052; Beyotime, China) following the manufacture's protocols. The absorbance of SOD and GSH was assessed at $450 \mathrm{~nm}$ and $412 \mathrm{~nm}$ respectively using a microplate reader and the SOD and GSH levels in cell homogenates were normalized to total cell protein.

\subsection{Mitochondrial DNA Quantification}

Total DNA was extracted from M-1 cells using MiniBEST Universal Genomic DNA Extraction Kit (TaKaRa, 9765) according to the manufacturers protocols. The number of mtDNA copies was quantified using real-time PCR with TB Green ${ }^{\circledast}$ Premix Ex Taq ${ }^{\text {tm }}$ II (TaKaRa, RR820) and a quantitative real time-PCR system (ABI, QuantStudio3). The primers specific for mitochondrion DNA and nuclear gene B2M were listed in Table 1 . The relative abundance of mtDNA copies was obtained by normalization to B2M using $2^{-\Delta \Delta \mathrm{Ct}}$ method.

Table 1. Primers used in this study.

\begin{tabular}{ccc}
\hline Primers & Sequences & Accession \\
\hline mMitoF1 & CTAGAAACCCCGAAACCAAA & NC_005089.1 \\
mMitoR1 & CCAGCTATCACCAAGCTCGT & NC_005089.1 \\
mB2MF1 & ATGGGAAGCCGAACATACTG & NC_000068.7 \\
mB2MR1 & CAGTCTCAGTGGGGGTGAAT & NC_000068.7 \\
\hline
\end{tabular}




\subsection{Transcriptome Sequencing}

Total RNA was extracted from the M-1 cells using the MiniBEST Universal RNA Extraction Kit (TaKaRa, 9767). The RNA was prepared from the cells treated with high and low doses of Astragali radix. The RNA samples were sent to Sangon Biotech (Shanghai) Co., Ltd. for transcriptome sequencing using the Illumina HiseqTM sequencing system.

\subsection{Statistical Analysis}

Values of the different measurements were normalized to a respective mean control value from untreated samples and expressed as percent control. All data is expressed as mean \pm standard deviation (SD). They were analysed using analysis of variance (ANOVA) and Least Significant Difference (LSD) using GraphPad InStat software, where $\mathrm{p}<0.05$ was considered statistically significant.

\section{Results}

\subsection{The Effect of Three Herbs on the Generation of ATP in M-1 Cells}

All three herbs showed a concentration-dependent effect on the generation of ATP, following a hormetic curve. As the concentration of the extracted Astragali radix and Herba cistanche increased from $12.5-100 \mu \mathrm{g} / \mathrm{mL}$, the total ATP content of M-1 cells also increased and was significantly higher than that of the control, and then dropped back to the same level as control at the high concentration of $200 \mu \mathrm{g} / \mathrm{mL}$ (upper panels of Figure 1(A) and Figure 1(B)); for Panax ginseng, as the concentration increased from $12.5-200 \mu \mathrm{g} / \mathrm{mL}$, ATP levels also gradually increased, peaked at the concentration of $25 \mu \mathrm{g} / \mathrm{mL}$, then decreased to a level that's significantly lower than the control at the high concentration of 200 $\mu \mathrm{g} / \mathrm{mL}$ (upper panel of Figure $1(\mathrm{C})$ ). However, the three herbs had no significant effect on the growth of M-1 cells at the concentrations tested as indicated by the lower panels of Figures 1(A)-(C). Based on these results, two concentrations of Astragali radix were chosen for further study: the low concentration of 50 $\mu \mathrm{g} / \mathrm{mL}$, within the hormetic zone, and the high concentration of $250 \mu \mathrm{g} / \mathrm{mL}$, outside the hormetc zone.

\subsection{The Effect of Astragali radix on Mitochondrial Functions, Mitochondrial Copy Numbers, Antioxidant Capacity, and Cell Growth}

We further investigated the effects of two different concentrations of Astragali radix on mitochondrial functions including ATP generation, ROS production, and MMP, as well as mitochondrial copy numbers. As shown in Figure 2(A), ATP was elevated at the low concentration treatment, but depressed at the high concentrations significantly at $4 \mathrm{~h}$, and the effect for both concentrations was time-dependent. As a by-product of ATP production, the total ROS for the low concentration showed no significant increase at $4 \mathrm{~h}$ and $24 \mathrm{~h}$ but a significant 

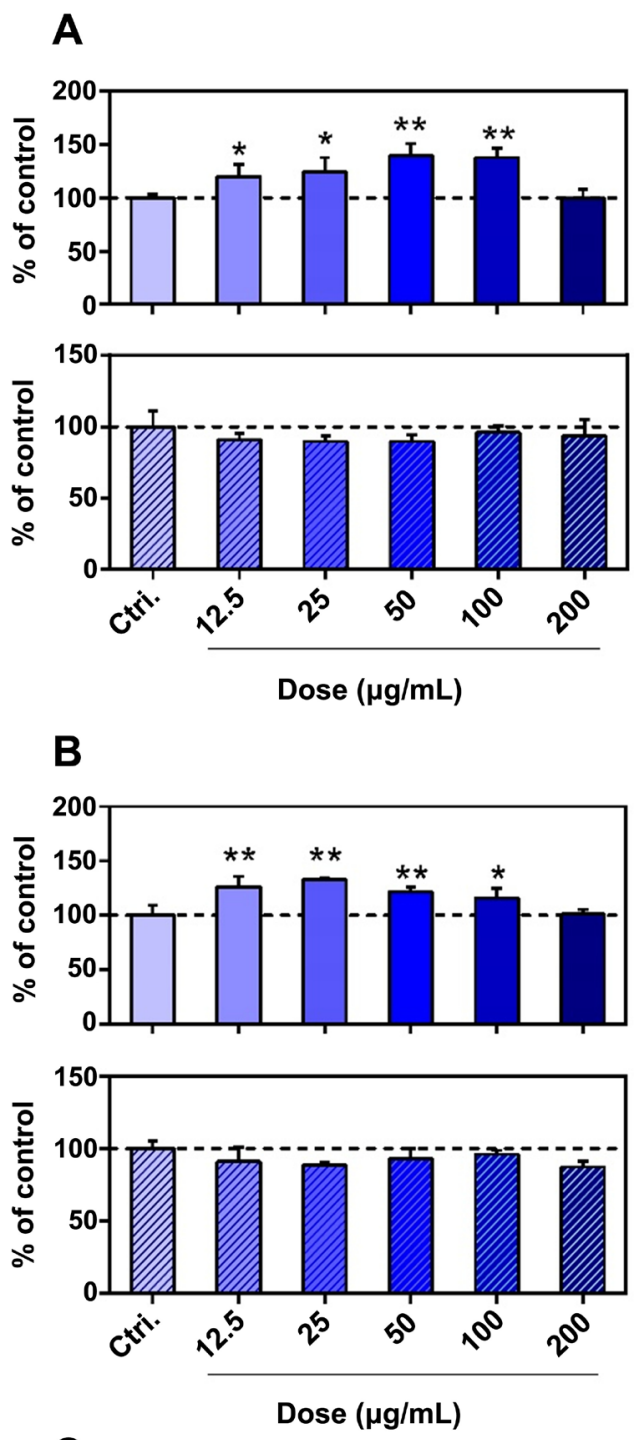

C
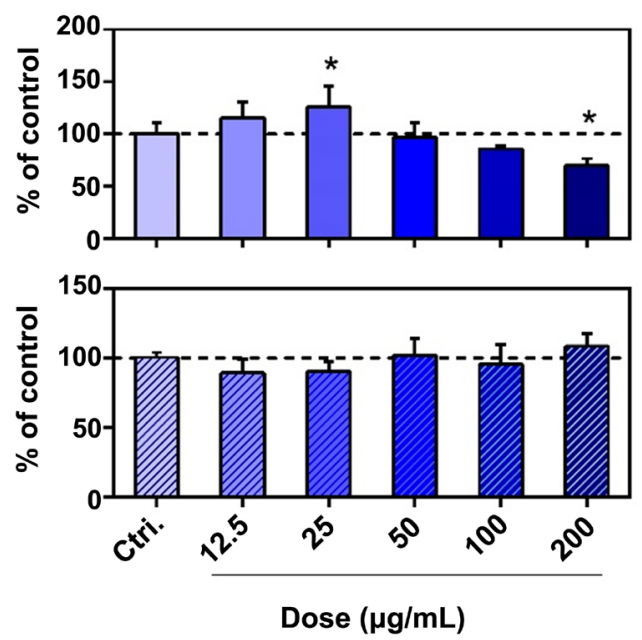

Figure 1. The effect of different TCM extract on mitochondrial ATP content (upper panel) and cell growth in M-1 cells (lower panel). (A) Astragali radix; (B) Herba cistanche; (C) Panax ginseng. ${ }^{*} \mathrm{P}<0.05 ;{ }^{*} \mathrm{P}<0.01$, when compared with the control. 

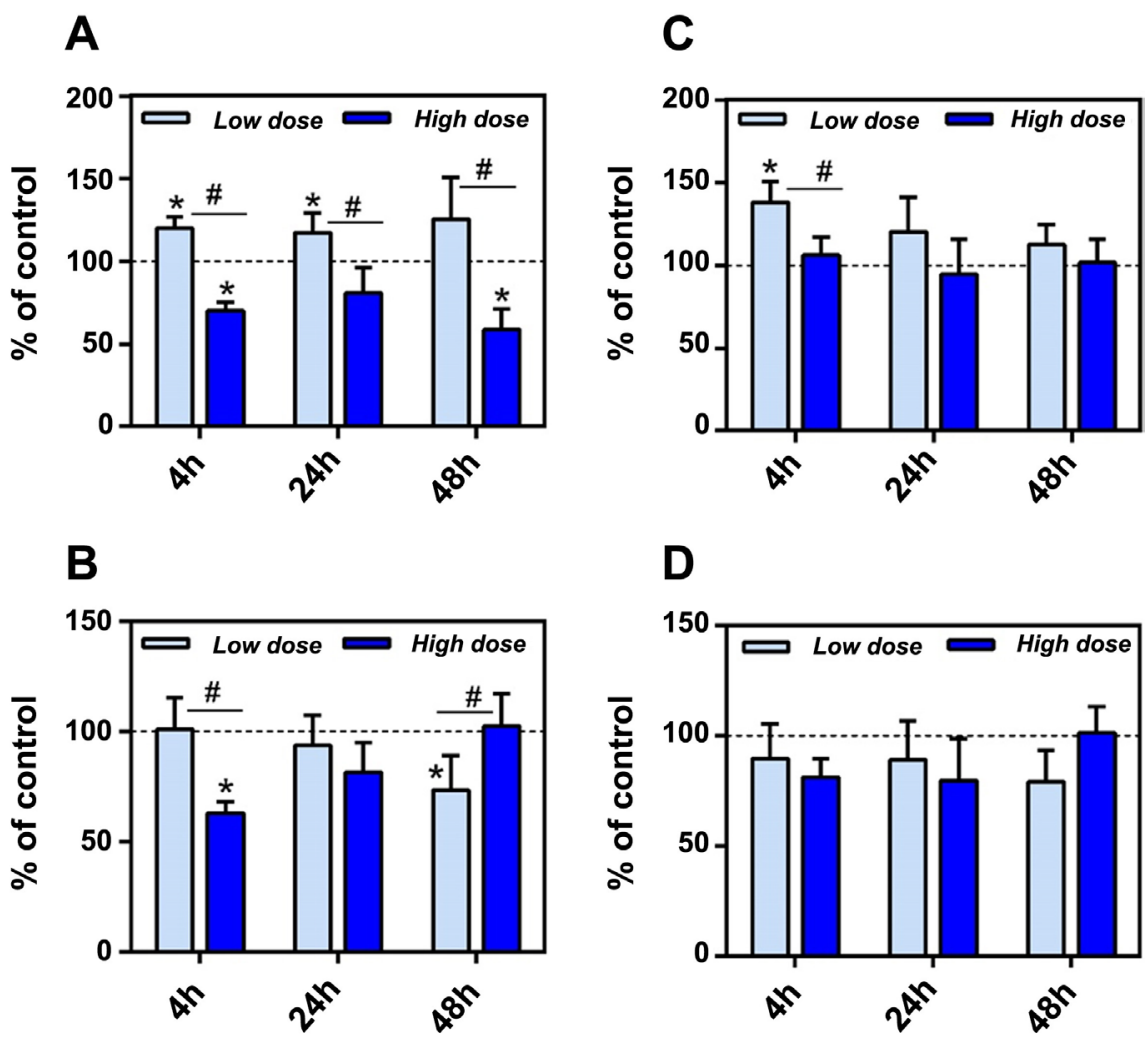

Figure 2. The effect of Astragali radix extract on mitochondrial function in M-1 cells. (A) ATP; (B) ROS; (C) MMP; (D) Mitochondrial copies. ${ }^{*}$ Significantly different from the control group; \#significantly different between the low dose and high dose treatment.

decrease at $48 \mathrm{~h}$, despite the fact that ATP increased significant at $4 \mathrm{~h}$ and $24 \mathrm{~h}$ (Figure 2(B)). Thus, for low concentrations of Astragali radix, an increased antioxidant capacity occurred to remove the excess ROS. However, at high concentrations the herb produced the opposite trend, with ROS significantly lower than the controls, but increased over time. At $4 \mathrm{~h}$, MMP was significantly increased for the low concentration, but was unchanged at the other times for either concentration (Figure 2(C)). Mitochondrial copy numbers were conserved with no significant changes observed for either treatment at all times.

At high concentrations of Astragali radix, the antioxidant capacity was depressed (Figure 3), with SOD and GSH being significantly lower at $24 \mathrm{~h}$ and 48 h, respectively. However, it is elevated for the low concentration group, with GSH significantly higher at $24 \mathrm{~h}$ and $48 \mathrm{~h}$. SOD was relatively conserved for the low concentration group. Despite the biochemical variations for the two concentrations, the growth of cells was not affected at all times for both concentrations as shown in Figure 4.

\subsection{The Effect of Astragali radix on Cell Gene Expression}

Genes that changed in the Astragali radix treatment group were selected when the following conditions were met: $\log _{2}$ (Fold Change) $>1$ or $\log _{2}$ (Fold Change $<-1$ and $p$ values $<0.05$. Comparing the transcriptome results for the 


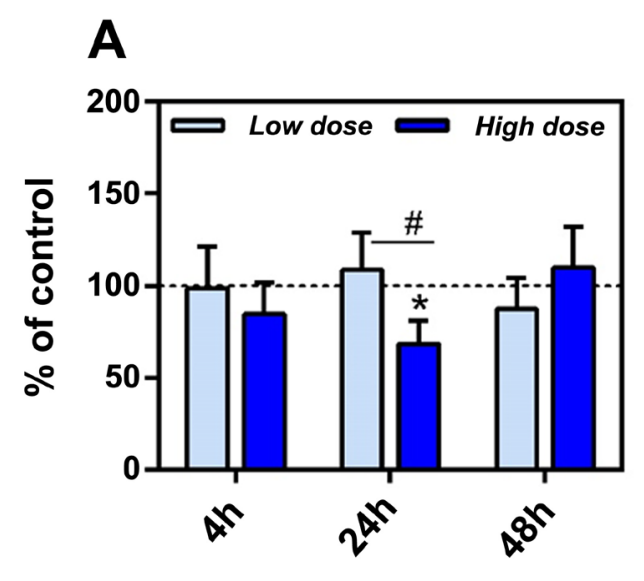

\section{B}

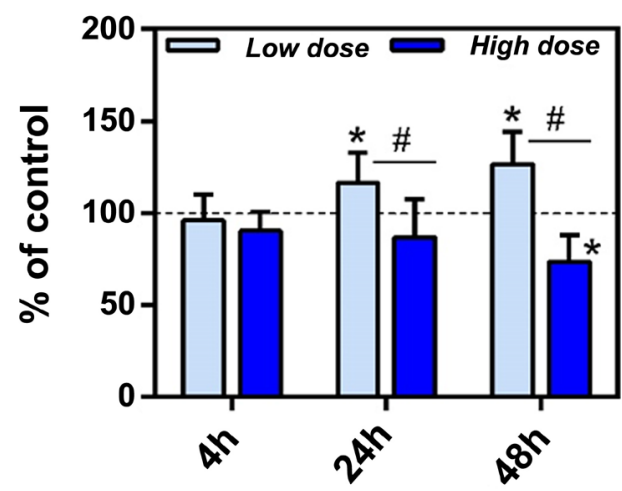

Figure 3. The effect of Astragali radix extract on antioxidant capacity in M-1 cells. (A) SOD; (B) GSH. *Significantly different from the control group; \#significantly different between the low dose and high dose treatment.

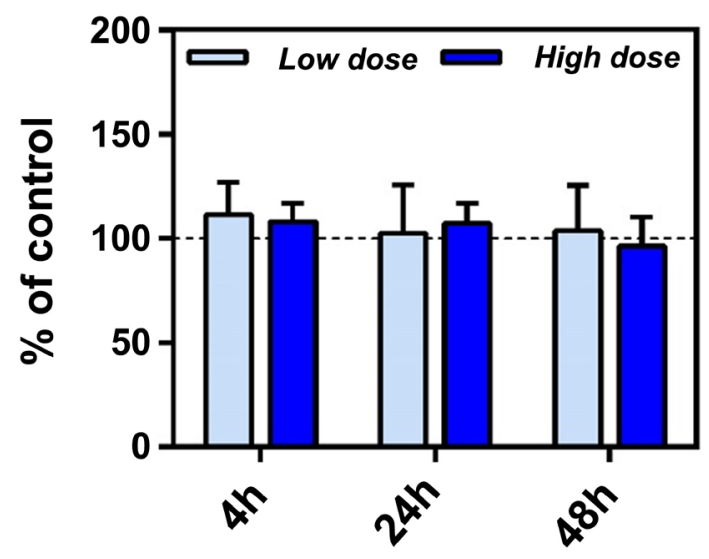

Figure 4. The effect of Astragali radix extract on cell growth in M-1 cells. ${ }^{*}$ Significantly different from the control group; \#significantly different between the low dose and high dose treatment.

two concentrations of Astragali radix, it was found that the expression levels of four genes have significantly changed for the treatment group as seen in Table 2. Among the four genes significantly changed, the expression level of NOCT gene 
Table 2. Genes significantly changed based on the transcriptome analysis.

\begin{tabular}{cccc}
\hline Gene ID & $\begin{array}{c}\text { Gene } \\
\text { Name }\end{array}$ & $\begin{array}{c}\text { High concentration } \\
\text { of } \text { Astragali radix }\end{array}$ & $\begin{array}{c}\text { Low concentration } \\
\text { of } \text { Astragali radix }\end{array}$ \\
\hline ENSMUSG00000023087 & Noct & Down & Up \\
ENSMUSG00000036214 & Znrd1as & Down & Down \\
ENSMUSG00000053128 & Rnf26 & Down & Down \\
ENSMUSG00000037533 & Rapgef6 & Up & Down \\
\hline
\end{tabular}

was upregulated in the low concentration group and downregulated in the high concentration group. The NOCT gene encodes the Nocturnin protein which has phosphatase activity, catalysing $\mathrm{NADP}^{+}$to $\mathrm{NAD}^{+}$or NADPH to NADH [27]. $\mathrm{NADH}$, as a carrier and electron donor of hydrogen, affects the level of mitochondrial ATP generation during the oxidative phosphorylation process. Transcriptome results showed that low-concentrations of Astragali radix promoted NOCT expression at $4 \mathrm{~h}$, while high-concentration Astragali radix inhibited NOCT expression at $4 \mathrm{~h}$. This result is consistent with the regulation of mitochondrial ATP levels by high- and low-concentration Astragali radix (Figure 2(A)), indicating that different concentrations of Astragali radix may affect the production of ATP by regulating its NADH levels through NOCT.

\section{Discussion}

In Traditional Chinese Medicine (TCM), Qi is defined as the life force energy within our bodies and the energy around us. However, current technology does not allow us to capture or measure Qi, despite its profound importance in TCM as the most fundamental concept. For different types of Qi, kidney is the root of vitality (Yuan Qi), which is considered the innate foundation of all biological systems. So, for this study, the mouse renal collecting duct cells were selected as the target to explore the cellular and molecular changes after treatment with Qi-invigorating traditional Chinese herbs.

Astragali radix, Panax ginseng and Herba cistanche are commonly used clinically as tonifying herbs, with the first two being Qi-invigorating and the last one Yang-invigorating. They are used for tonifying Qi and promoting Yang, with their efficacy well documented in the classical TCM text, the Shennong Herbal Classic. However, its mechanism is not well understood from a western medical perspective.

The linkage between mitochondria and Qi/Chi was first introduced by Dr. Wallace [4], who proposed that TCM herbs would cure mitochondria related diseases. Based on our current data, Qi-invigorating herbs not only enhanced ATP production, but also boosted antioxidant capacity in kidney cells. As a result, the cells are exposed to less oxidative stress while maintaining a high energy output. We speculate that the enhanced energy output is used for repair-related cell functions, as cell growth was unchanged. This is consistent with the general functions of Qi to increase vitality and immunity, as well as safeguarding all bi- 
ological systems [4] [28].

Ko et al. [29] examined the biochemical basis of Yang-invigorating and Yin-nourishing herbs and concluded that Yang-invigorating herbs boost ATP generation while Yin-nourishing herbs enhance immunomodulatory functions ex vivo or in $\mathrm{H} 9 \mathrm{C} 2$ cardiomyocytes in vitro [7]. The results reported here are consistent with the findings of Ko et al., as all three herbs enhanced ATP generation in a concentration and time-dependent manner. It was found that with increasing concentrations of Qi-invigorating herbs, the total ATP content of M-1 cells first increased and then decreased. Thus, Astragali radix showed an inverted U-shaped dose-response curve, whereas Panax ginseng and Herba cistanche showed a J-shaped dose-response curve consistent with the theory of hormesis. Hormesis is an adaptive response to stress which exhibits a biphasic curve characterized by excitation at low doses and inhibition at high doses, and according to the different end points generates an inverted U-shaped or J-shaped dose-response curve [30].

Since Astragali radix extract showed a significant effect on M-1 cells, we used it for a more detailed study, with a focus on mitochondrial functions and antioxidant capacity of the cells. For the sake of the robustness of the results, two concentrations of Astragali radix were selected with one in and the other out of the hormetic zone. The low dose of Astragali radix induced an adaptive response that resulted in an increased antioxidant capacity and an increase in mitochondrial energetic status, promoting cellular health benefits.

Both concentrations of Astragali radix had no significant effect on cell growth during the time tested. However, numerous changes were observed at both the biochemical and molecular level. The low concentration of the Qi-invigorating herbs increased ATP production which is normally associated with a higher production of ROS. However, our data indicate that under none of the experimental conditions did Astragali radix increase ROS levels, but in fact reduced it significantly. This indicates that the antioxidant capacity was enhanced by the Qi-invigorating herb to support the continued increase in ATP production. This interpretation is supported by the observed enhancement of GSH at $24 \mathrm{~h}$ and 48 $\mathrm{h}$, but not at $4 \mathrm{~h}$ indicating it was an induced response to the increase in ATP and ROS. The current results are inconsistent with a recent study using H9C2 cells, which showed that Qi-invigorating herbs boost ATP production mainly because of enhancement by reduced GSH, which preserves the function of the mitochondria [31]. More research needs to be done regarding the mechanism of the increase in GSH by Qi-invigorating herbs to better understand the relationship between the increase in ATP and GSH.

Mitochondrial membrane potential $(\Delta \Psi \mathrm{m})$ is generated by the proton pump which is a critical parameter responsible for ATP synthesis, calcium accumulation and the maintenance of mitochondrial shape [32]. In our study, MMP was only increased at $4 \mathrm{~h}$, but dropped back to normal level at $24 \mathrm{~h}$ and $48 \mathrm{~h}$ despite the elevated ATP production, indicating it is a relatively conservative parameter. This is consistent with the findings by Zorova et al. who points out that it is crit- 
ical for cells to maintain the stability of MMP levels because the rise or drop of MMP will lead to unwanted loss of cell vitality, causing various pathologies [33]. The amount of mtDNA is associated with mitochondrial function, which varies with a number of cellular and environmental factors [34]. In this study, the number of mtDNA copies did not change upon the treatment of Astragali radix at both concentrations.

As expected, the high concentration on the other hand reduced the production of ATP, ROS and the antioxidant capacity as indicated by SOD and GSH activities. Wang et al. [35] proposed that the stimulatory effect at low doses and inhibitory effect at high does on a hormetic curve correspond to the regulatory and curing mode of the TCM herbs. The regulatory effect induces adaptive responses and restores the Yin-Yang balance, while the curing action is to prevent the manifestation of disease. In our study, the low-dose "regulatory" effect is to boost the ATP as well as the GSH-mediated antioxidant capacity, while the "curing" effect is to slow down the energy metabolism as evidenced by the reduced ATP and ROS production.

The molecular mechanism for the metabolic effects of Qi-invigorating herbs is not well understood. In our study, by conducting a transcriptome analysis, we identified four genes that are significantly affected by Astragali radix, among which the NOCT gene which is responsible for the conversion of NADPH to $\mathrm{NADH}$ is of special interest. Low concentration of Astragali radix promoted NOCT expression, while high concentration inhibited NOCT expression. The upregulated NOCT gene expression leads to the increase of its catalytic product $\mathrm{NADH}$, which in turn increases the efficiency of the electron transfer in the respiratory transport chain ultimately leading to the increase of ATP production. Therefore, NOCT gene may play an important role in the function of Astragali radix in regulating the function of mitochondria. This study lays the foundation for further exploring the molecular mechanisms of Qi-invigorating herbs. Through the study of the effect of Qi-invigorating herbs on the mitochondrial functions, we hope to understand Qi from the perspective of modern sciences. According to Dr. Wallace, "TCM and western medicine will meet at mitochondria."

\section{Conclusion}

We found that, in cultured cells, Qi-invigorating herbs can enhance the function of mitochondria including ATP production and antioxidant capacity, which yielded reduced ROS levels. This indicates that the biochemical basis of Qi-invigorating herbs is to boost the energy production of the mitochondria while reducing the ROS by enhancing the antioxidant capacity. Despite all the changes in mitochondria, the cell growth was not affected, indicating a role for mitochondria in maintaining cellular homeostasis. Since this effect also occurred for NOCT gene expression, it could explain that the increase in ATP generation was caused by a more efficient turnover of the NADH. This work for the first 
time examined both the biochemical and molecular basis of the Qi-invigorating herbs, with the goal of providing new insights into the mechanisms of TCM herbs from a western perspective.

\section{Acknowledgements}

The authors would like to thank the ENN Research Fund.

\section{Conflicts of Interest}

The authors declare no conflicts of interest regarding the publication of this paper.

\section{References}

[1] Li, X.T. and Zhao, J. (2012) An Approach to the Nature of Qi in TCM-Qi and Bioenergy. In: Kuang, H.X., Ed., Recent Advances in Theories and Practice of Chinese Medicine, IntechOpen, London, 79-108. https://doi.org/10.5772/28416

[2] Yao, W., Yang, H. and Ding, G. (2013) Mechanisms of Qi-Blood Circulation and Qi Deficiency Syndrome in View of Blood and Interstitial Fluid Circulation. Journal of Traditional Chinese Medicine, 33, 538-544. https://doi.org/10.1016/S0254-6272(13)60162-4

[3] Cornelius, C., Perrotta, R., Graziano, A., Calabrese, E.J. and Calabrese, V. (2013) Stress Responses, Vitagenes and Hormesis as Critical Determinants in Aging and Longevity: Mitochondria as a "Chi”. Immunity \& Ageing, 10, Article No. 15. https://doi.org/10.1186/1742-4933-10-15

[4] Wallace, D.C. (2008) Mitochondria as Chi. Genetics, 179, 727-735. https://doi.org/10.1534/genetics.104.91769

[5] Zhang, M.L., Zhang, L.T., Qiu, X.F. and Zhou, A.F. (2001) Discussion on the Relationship of Mitochondrion and Qi. Chinese Journal of Basic Medicine in Traditional Chinese Medicine, 7, 60-61.

[6] Lin, F., Guo, L.L. and Wang, J. (2014) Expounding the Functions of Qi in TCM Based on the Effect of Mitochondria. Chinese Journal of Integrated Traditional and Western Medicine, 34, 903-905.

[7] Wong, H.S., Cheung, W.F., Tang, W.L. and Ko, K.M. (2012) “Qi-Invigorating” Chinese Tonic Herbs (Shens) Stimulate Mitochondrial ATP Generation Capacity in H9c2 Cardiomyocytes in Situ and Rat Hearts ex Vivo. Chinese Medicine, 3, 101-105. http://dx.doi.org/10.4236/cm.2012.32016

[8] Chen, J., Wong, H.S., Leong, P.K., Leung, H.Y., Chan, W.M. and Ko, K.M. (2014) New Insights into the Chemical and Biochemical Basis of the "Yang-Invigorating" Action of Chinese Yang-Tonic Herbs. Evidence-Based Complementary and Alternative Medicine, 2014, Article ID: 856273. https://doi.org/10.1155/2014/856273

[9] Ko, K.M. and Chiu, P.Y. (2006) Biochemical Basis of the "Qi-Invigorating" Action of Schisandra Berry (Wu-Wei-Zi) in Chinese Medicine. The American Journal of Chinese Medicine, 34, 171-176. https://doi.org/10.1142/S0192415X06003734

[10] Wallace, D.C. (2012) Mitochondria and Cancer. Nature Reviews Cancer, 12, 685-698. https://doi.org/10.1038/nrc3365

[11] Picard, M., Wallace, D.C. and Burelle, Y. (2016) The Rise of Mitochondria in Medicine. Mitochondrion, 30, 105-116. https://doi.org/10.1016/j.mito.2016.07.003

[12] Srivastava, S. (2017) The Mitochondrial Basis of Aging and Age-Related Disorders. 
Genes, 8, 398-421. https://doi.org/10.3390/genes8120398

[13] Picard, M. and Mcewen, B.S. (2018) Psychological Stress and Mitochondria: A Conceptual Framework. Psychosomatic Medicine, 80, 126-140. https://doi.org/10.1097/PSY.0000000000000544

[14] Zhang, Q. (2018) Effect Analysis of Buqi Changluo Decoction in Treating Chronic Heart Failure Patients with Coronary Heart Disease. Clinical Journal of Chinese Medicine, 10, 36-37

[15] Liu, X.D., Li, J.L., Liang, T. and Lu, L. (2019) Clinical Effect of Buqi Gutuo Chinese Medicine on Patients with Unstable Angina Pectoris. Clinical Journal of Chinese Medicine, 11, 18-19. http://dx.chinadoi.cn/10.3969/j.issn.1674-7860.2019.24.008

[16] Chu, X.Z. and Yang, L. (2019) Clinical Study on the Therapeutic Effect of Nourishing Qi, Activating Blood and Reinforcing Water on Chronic Heart Failure of Qi Deficiency and Blood Stasis Type. Electronic Journal of Clinical Medical Literature, 6, 32-34.

[17] Li, H.J. (2019) Effect of Buqi Yunpi Decoction on Traditional Chinese Medicine Syndrome Scores, Negative Conversion of Helicobacter Pylori and Adverse Reactions for Patients with Chronic Gastritis of Spleen-Stomach Deficiency. Journal of Sichuan of Traditional Chinese Medicine, 37, 128-130.

[18] Jiang, Y.H. (2019) Study on the Effect of Wenzhong Buqi Prescription on Gastric Cancer Patients and the Change of $\mathrm{CD}^{+} \mathrm{CD} 4^{+} \mathrm{CD}^{+} / \mathrm{CD}^{+}$. Chinese Archives of Traditional Chinese Medicine, 10, 1-6.

[19] Zhao, J.J. (2018) Clinical Study of Wenzhong Buqi Decoction in Treating for Gastric Cancer with Spleen-Stomach Cold Deficiency Syndrome. Acta Chinese Medicine, 33, 1865-1869.

[20] Luo, N.X., Qin, Q., Gong, W.J. and Li, H. (2018) Clinical Effect of Buqihuoxuefa on Postoperative Recurrence of Ovarian Endometrial Cyst. Popular Science \& Technology, 20, 38-41.

[21] Leong, P.K. and Ko, K.M. (2018) Shengmai San: A Modern Medicine Perspective on Its Remedial Effects on Qi and Yin Deficiency Syndrome in Chinese Medicine. Longhua Chinese Medicine, 1, Article No. 13. https://doi.org/10.21037/lcm.2018.09.03

[22] Ko, K.M., Mak, D.H., Chiu, P.Y. and Poon, M.K. (2004) Pharmacological Basis of 'Yang-Invigoration' in Chinese Medicine. Trends in Pharmacological Sciences, 25, 3-6. https://doi.org/10.1016/j.tips.2003.11.002

[23] Ko, K.M. and Leung, H.Y. (2007) Enhancement of ATP Generation Capacity, Antioxidant Activity and Immunomodulatory Activities by Chinese Yang and Yin Tonifying Herbs. Chinese Medicine, 2, Article No. 3.

https://doi.org/10.1186/1749-8546-2-3

[24] Leung, H.Y., Chiu, P.Y., Poon, M.K. and Ko, K.M. (2005) A Yang-Invigorating Chinese Herbal Formula Enhances Mitochondrial Functional Ability and Antioxidant Capacity in Various Tissues of Male and Female Rats. Rejuvenation Research, 8, 238-247. https://doi.org/10.1089/rej.2005.8.238

[25] Wong, H.S., Leung, H.Y. and Ko, K.M. (2011) 'Yang-Invigorating' Chinese Tonic Herbs Enhance Mitochondrial ATP Generation in H9c2 Cardiomyocytes. Chinese Medicine, 2, 1-5. http://dx.doi.org/10.4236/cm.2011.21001

[26] Chen, Y.J., Wu, X.X. and Li, T.J. (2018) A Method for Detecting the Energy of Organism. China Patent No. CN108303475A.

[27] Estrella, M.A., Du, J., Chen, L., Rath, S., Prangley, E., Chitrakar, A., Aoki, T., Schedl, 
P., Rabinowitz, J. and Korennykh, A. (2019) The Metabolites $\mathrm{NADP}^{+}$and NADPH Are the Targets of the Circadian Protein Nocturnin (Curled). Nature Communications, 10, Article No. 2367. https://doi.org/10.1038/s41467-019-10125-Z

[28] Lin, F., Guo, L. and Wang, J. (2014) Discussion on the Relationship of Mitochondrion and Qi. Chinese Journal of Basic Medicine in Traditional Chinese Medicine, 34, 903-906.

[29] Ko, K.M., Leon, T.Y.Y., Mak, D.H.F., Chiu, P.Y., Du, Y. and Tpoon, M.K. (2006) A Characteristic Pharmacological Action of 'Yang-Invigorating' Chinese Tonifying Herbs: Enhancement of Myocardial ATP-Generation Capacity. Phytomedicine, 13, 636-642. https://doi.org/10.1016/j.phymed.2006.02.007

[30] Leong, P.K. and Ko, R.K.M. (2018) Hormesis: Mechanistic Implications in Herbal Treatment in Traditional Chinese Medicine. Longhua Chinese Medicine, 1, Article No. 4. http://dx.doi.org/10.21037/lcm.2018.04.01

[31] Leong, P.K., Leung, H.Y., Chan, W.M. and Ko, K.M. (2018) Differences in the Mechanisms by Which Yang-Invigorating and Qi-Invigorating Chinese Tonifying Herbs Stimulate Mitochondrial ATP Generation Capacity. Chinese Medicine, 9, 63-74. https://doi.org/10.4236/cm.2018.92005

[32] Ma, X.Y., Deng, D. and Chen, W.D. (2017) Inhibitors and Activators of SOD, GSH cc x, and CAT. In: Şentürk, M., Ed., Enzyme Inhibitors and Activators, IntechOpen, London, 207-224. https://doi.org/10.5772/65936

[33] Zorova, L.D., Popkov, V.A., Plotnikov, E.Y., Silachev, D.N., Pevzner, I.B., Jankauskas, S.S., Babenko, V.A., Zorov, S.D., Balakireva, A.V. and Juhaszova, M. (2017) Mitochondrial Membrane Potential. Analytical Biochemistry, 552, 50-59. https://doi.org/10.1016/j.ab.2017.07.009

[34] Ajaz, S., Czajka, A. and Malik, A. (2015) Accurate Measurement of Circulating Mitochondrial DNA Content from Human Blood Samples Using Real-Time Quantitative PCR. In: Weissig, V. and Edeas, M., Eds., Mitochondrial Medicine, Vol. 1264. Humana Press, New York. https://doi.org/10.1007/978-1-4939-2257-4_12

[35] Wang, D., Calabrese, E.J., Lian, B., Lin, Z. and Calabrese, V. (2018) Hormesis as a Mechanistic Approach to Understanding Herbal Treatments in Traditional Chinese Medicine. Pharmacology \& Therapeutics, 184, 42-50. https://doi.org/10.1016/j.pharmthera.2017.10.013 